\title{
Efficacy of treating pain to reduce behavioural disturbances in residents of nursing homes with dementia: cluster randomised clinical trial
}

\author{
Bettina S Husebo postdoctoral fellow ${ }^{1}$, Clive Ballard professor ${ }^{2}$, Reidun Sandvik registered nurse ${ }^{1}$, \\ Odd Bjarte Nilsen statistician ${ }^{3}$, Dag Aarsland professor ${ }^{4}$
}

1'Department of Public Health and Primary Health Care, University of Bergen, 5020 Bergen, Norway; ${ }^{2}$ Wolfson Centre for Age-Related Diseases, Wolfson Wing and Hodgkin Building, Guy's Campus, Kings College, London SE1 1UL, UK; ${ }^{3}$ Department of Psychiatry, Stavanger University Hospital, 4011 Stavanger, Norway; ${ }^{4}$ Karolinska Institute, Department of Neurobiology, Care Sciences and Society, Karolinska Institute-Alzheimer Disease Research Center, Novum, Stockholm, Stavanger University Hospital, Department of Psychiatry, Stavanger, Norway, and University of Oslo, Oslo, Norway

\begin{abstract}
Objective To determine whether a systematic approach to the treatment of pain can reduce agitation in people with moderate to severe dementia living in nursing homes.

Design Cluster randomised controlled trial.

Setting 60 clusters (single independent nursing home units) in 18 nursing homes within five municipalities of western Norway.

Participants 352 residents with moderate to severe dementia and clinically significant behavioural disturbances randomised to a stepwise protocol for the treatment of pain for eight weeks with additional follow-up four weeks after the end of treatment ( 33 clusters; $n=175$ ) or to usual treatment (control, 27 clusters; $\mathrm{n}=177$ ).
\end{abstract}

Intervention Participants in the intervention group received individual daily treatment of pain for eight weeks according to the stepwise protocol, with paracetamol (acetaminophen), morphine, buprenorphine transdermal patch, or pregabaline. The control group received usual treatment and care.

Main outcome measures Primary outcome measure was agitation (scores on Cohen-Mansfield agitation inventory). Secondary outcome measures were aggression (scores on neuropsychiatric inventory-nursing home version), pain (scores on

mobilisation-observation-behaviour-intensity-dementia-2), activities of daily living, and cognition (mini-mental state examination).

Results Agitation was significantly reduced in the intervention group compared with control group after eight weeks (repeated measures analysis of covariance adjusting for baseline score, $\mathrm{P}<0.001$ ): the average reduction in scores for agitation was $17 \%$ (treatment effect estimate $-7.0,95 \%$ confidence interval -3.7 to -10.3 ). Treatment of pain was also significantly beneficial for the overall severity of neuropsychiatric symptoms $(-9.0,-5.5$ to -12.6$)$ and pain $(-1.3,-0.8$ to -1.7$)$, but the groups did not differ significantly for activities of daily living or cognition.
Conclusion A systematic approach to the management of pain significantly reduced agitation in residents of nursing homes with moderate to severe dementia. Effective management of pain can play an important part in the treatment of agitation and could reduce the number of unnecessary prescriptions for psychotropic drugs in this population.

Trial registration ClinicalTrials.gov NCT01021696 and Norwegian Medicines Agency EudraCTnr 2008-007490-20.

\section{Introduction}

Thirty five million people worldwide have dementia, and this number is expected to increase to 115 million by $2050 .{ }^{1}$

Agitation and aggression are common in people with dementia, in particular those with moderate to severe dementia living in nursing homes, where the cross sectional prevalence of these symptoms exceeds 50\%. ${ }^{23}$ Agitation is associated with increased distress to residents and a burden to family and professional caregivers ${ }^{4}$ and is one of the most challenging symptoms for clinical management.

Antipsychotics are often used as first line drug treatment for agitation and aggression, with $40-60 \%$ of residents with dementia in nursing homes prescribed such treatment. ${ }^{5}$ In the United Kingdom alone, a report for the Department of Health estimated that 180000 people with dementia were being prescribed antipsychotics, causing 1620 excess strokes and 1800 deaths a year. ${ }^{6}$ These figures emphasise the importance of finding safe and effective ways to reduce agitation and aggression in people with dementia.

Many people with dementia have painful conditions, ${ }^{7}$ and it has been proposed that pain in patients with impaired language and abstract thinking may manifest as agitation. ${ }^{8}$ Thus more effective treatment of undiagnosed pain may contribute to the overall prevention and management of agitation. Overall, 50-80\% of 
residents in nursing homes are affected by pain ${ }^{9}$ and most good practice guidelines emphasise the importance of pain management in the treatment of neuropsychiatric symptoms in people with dementia. ${ }^{10}$ Few studies have, however, explored the potential utility of treating pain as a way of improving agitation. ${ }^{11-14}$ We evaluated whether systematic use of analgesics can reduce agitation in residents of nursing homes with moderate to severe dementia.

\section{Methods}

During October 2009 to June 2010 we carried out a multicentre, cluster randomised controlled trial for eight weeks, with an additional follow-up at 12 weeks (four weeks after the end of treatment) in 60 nursing home units within five municipalities of western Norway. A cluster was defined as a single independent nursing home unit (with no crossover of staff). We chose this design primarily to avoid contamination, because care staff receiving training in the assessment and treatment of pain cannot be expected to treat individual residents differently. The study statistician $(\mathrm{OBN})$ used Stata version 8 to generate a list of random numbers for allocation of clusters to one of the two groups.

Eligible participants were adults aged 65 or more, living in one of the nursing homes for at least four weeks, with dementia according to Diagnostic and Statistical Manual of Mental Disorders, fourth edition, functional assessment staging score of more than 4, and clinically relevant behavioural disturbances, defined as a score of 39 or more on the Cohen-Mansfield Agitation Inventory - that is, clinically significant agitation for at least one week. ${ }^{15}$ Exclusion criteria were advanced severe medical disease with expected survival of less than six months, severe psychiatric or neurological disorder, severe aggression (agitation score $\geq 8$ on the neuropsychiatric inventory-nursing home version, ${ }^{16}$ with aggression as the predominant symptom), severe liver or renal failure, severe injury or anaemia (haemoglobin concentration $<8.5 \mathrm{mmol} / \mathrm{L}$ ), and known allergy to paracetamol (acetaminophen), morphine, buprenorphine, or pregabaline.

\section{Intervention}

We randomly assigned patients to receive either treatment for pain according to a stepwise protocol for eight weeks or their usual management (the control group). The stepwise protocol followed the recommendations of the American Geriatrics Society. ${ }^{17}$ Participants in the intervention group received analgesics according to the standardised protocol. Depending on the ongoing medical treatment, participants allocated to the treatment protocol started at step 1 (oral paracetamol, maximum increase to $3 \mathrm{~g} /$ day) or, if they were already receiving treatment were adjusted to either step 2 (oral morphine, maximum 20 $\mathrm{mg} /$ day), step 3 (buprenorphine transdermal patch, maximum $10 \mu \mathrm{g} / \mathrm{h}$ ), or step 4 (oral pregabaline, maximum $300 \mathrm{mg} / \mathrm{day}$ ), using a fixed dose regimen throughout the eight week treatment period. Residents with swallowing difficulties were started at step 3. Drugs were offered at breakfast, lunch, and dinner (about 08:00, noon, 18:00), respectively. If needed, combination therapy was allowed. In those who were not able to tolerate this treatment, the dosage was either reduced or the participant was withdrawn from the study and treated as clinically appropriate.

\section{Concomitant drugs}

Anti-dementia drugs, psychotropics, aspirin (one dose daily), or anti-inflammatory drugs (for example, ibuprofen) were allowed if participants had remained stable on these for four weeks before study inclusion. We allowed the use of analgesics as needed (other than paracetamol) and monitored this during the study. Clinicians were advised to keep prescriptions and doses of psychotropics unchanged when possible.

\section{Primary and secondary outcome measures}

The primary outcome was agitation, as measured on the Cohen-Mansfield agitation inventory, a nurses' rating questionnaire consisting of 29 agitated behaviours, each rated on a 7 point scale of frequency $(1=$ not present, $7=$ several times an hour; range 29-203). ${ }^{15}$ Secondary outcome measures were aggression (neuropsychiatric inventory-nursing home version), ${ }^{16}$ pain

(mobilisation-observation-behaviour-intensity-dementia-2), ${ }^{18}$ cognition (mini-mental state examination), ${ }^{19}$ activities of daily living, ${ }^{20}$ and functional assessment staging. The

Cohen-Mansfield agitation inventory, neuropsychiatric inventory-nursing home version, and

mobilisation-observation-behaviour-intensity-dementia-2 pain scale were completed at baseline, at two, four, and eight weeks after baseline, and at four weeks after the conclusion of treatment. We evaluated cognition (mini-mental state examination), activities of daily living, functional assessment staging, and drugs at baseline and after eight weeks of treatment.

\section{Procedures}

After receiving specific training in use of the instruments two research assistants carried out assessments by interviewing the primary caregiver during enrolment. A consultant for old age psychiatry (DA), an anaesthetist and pain therapist (BSH), one of the research assistants (RS), and a senior member of staff from each nursing home reviewed the outcomes of assessment and drug prescriptions for each patient after completion of baseline assessment but before randomisation.

Research assistants and caregivers were blinded to group allocation during assessments of the primary and secondary outcomes. Staff members in direct care contact with participants were unaware of the type of intervention. To ensure blindness, researchers and nurses with responsibility for carrying out the intervention did not participate in data collection. Nursing home staff were instructed not to discuss management procedures.

Safety and tolerability were monitored at each assessment and all adverse events and vital signs recorded.

\section{Sample size}

Based on the magnitude of improvement in randomised controlled trials of non-drug interventions for agitation in residents of nursing homes, ${ }^{21}$ we estimated that a $25 \%$ greater reduction of Cohen-Mansfield agitation inventory score in the intervention group compared with control group would indicate equivalence to the best currently available approaches. For example, in a recent study we found that the largest improvement for a psychosocial intervention was 7.1 points, and thus a $25 \%$ difference would be about a 5 versus 7 point improvement in the two groups. ${ }^{22}$ To measure a difference of this magnitude would require a minimum of 81 patients allocated to each arm of the trial, for a significance level of 5\% (two sided), a power of $95 \%$, and equal allocation.

Cluster randomisation leads to loss of power. ${ }^{23}$ To retain power, the sample size should be multiplied by $1+(m-1) \rho$, called the design effect, where $m$ is the average cluster size and $\rho=s_{b}^{2} /\left(s_{b}^{2}+s^{2}{ }_{w}\right)$ is the intracluster correlation coefficient, where $\mathrm{s}^{2}{ }_{\mathrm{b}}$ is the variance between clusters and $\mathrm{s}_{\mathrm{w}}^{2}$ is the variance within clusters. Based on additional assumption of an estimated 
intracluster correlation coefficient of 0.13 , an average of seven eligible and consenting participants in each cluster, the formula gives a design effect of $1.78(1+(7-1) \times 0.13)$. Thus we determined that we needed a minimum of $169.1(1.78 \times 95)$ eligible participants per arm, which is in order of 26 clusters per arm, or 52 clusters with 338 participants in total. To allow for dropout of individual participants and loss of clusters, we aimed at including 60 clusters.

\section{Statistical analysis}

We used $\chi^{2}$ and Mann-Whitney tests as appropriate to compare personal and clinical characteristics between the two groups. The analysis of covariance was used to estimate the mean effect in each treatment arm, weighted across clusters according to the number of participants within each cluster, and from this we obtained the mean treatment effect estimate (difference between groups) at each time point. ${ }^{24}$ The intracluster correlation coefficient expressed the proportion of the total variance in the data due to the between cluster variability. The primary efficacy population included all residents with at least one post-baseline assessment, using the last observation carried forward procedure to account for missing values. Treatment effect was expressed as estimated effect of intervention, along with a $95 \%$ confidence interval and the $\mathrm{P}$ values for each time point. Additional analyses included repeated measurement analysis of covariance without the last observation carried forward and comparison of change at weeks 2, 4, and 8 using Student's $t$ test. We used the same procedures to analyse the secondary outcome measures of pain and aggression, whereas to analyse the effect on the secondary outcome measures of cognition and activities of daily living we used Student's $t$ test to compare the changes between baseline and week 8 between the two treatment groups. Analyses were carried out using predictive analytics software statistics 17 (SPSS; Chicago, IL).

\section{Results}

In total, 920 nursing homes residents were screened; 420 had moderate to severe dementia with behavioural disturbances and were assessed for eligibility. Sixty eight were excluded from randomisation because they did not meet the inclusion criteria, they declined to participate, or there were other reasons (fig 1). Overall, 352 residents in 60 clusters underwent randomisation ( 27 control clusters $(n=177), 33$ intervention clusters $(n=175)$, fig 1). The median number of participants in each cluster was 6.5 (range 2-11). The groups had similar personal and clinical characteristics and outcome measures at baseline (table 1). In all, $59 \%(n=103)$ of the intervention group and $55 \%(n=98)$ of the control group had clinically relevant pain scores of 3 or more on the mobilisation-observation-behaviour-intensity-dementia-2 pain scale at baseline.

During the eight weeks 20 participants were lost in the control group and 28 in the intervention group $(\mathrm{P}=0.298)$. Fourteen participants died during the study period, eight in the control group and six in the intervention group (fig 1).

\section{Analgesics during intervention}

Table 2 shows the distribution of treatments for pain. In the intervention group, $111(63 \%)$ participants received step 1 (paracetamol $3 \mathrm{~g} /$ day) of the treatment protocol, and in addition nine patients $(5 \%)$ an existing low dosage was increased. Four participants $(2 \%)$ received step 2 (three started with morphine; in one patient the primary prescription was adjusted). Thirty one participants $(18 \%)$ received step 3 (buprenorphine transdermal patch), and in addition eight participants (5\%) the dosage was increased. Twelve participants (7\%) received step 4 (pregabaline).

\section{Outcome}

Table 3 and figure 2 show the change in scores on the Cohen-Mansfield agitation inventory in the two groups. The repeated measurement analysis of covariance, using the population with last observation carried forward, was significantly different between the intervention and control groups after eight weeks, in favour of pain treatment $(\mathrm{P}<0.001)$ : the average reduction in agitation was $17 \%$ (treatment effect estimate $-7.0,95 \%$ confidence interval -3.7 to -10.3 ). The findings were similar when analysed based on only completers $(\mathrm{P}<0.001)$, and the change from baseline differed between the groups at weeks 2,4 , and 8 . In the intervention group there was a worsening of scores on the Cohen-Mansfield agitation inventory between week 8 and week 12, after withdrawal of pain treatment.

Between group differences in favour of pain treatment were also significant for aggression $(-9.0,-5.5$ to -12.6$)$ and pain $(-1.3,-0.8$ to -1.7 ; tables 4 and 5$)$. The correlation between pain and aggression was significant at week $8(\mathrm{P}=0.01)$. At week 8 the intervention and control groups did not differ significantly for cognition (mini-mental state examination) $(\mathrm{P}=0.127$; mean 7.4 and 8.6, respectively) or activities of daily living $(\mathrm{P}=0.443$; mean 7.9 and 8.4 , respectively).

\section{Discussion}

A standardised stepwise protocol of treatment with analgesics in residents of nursing homes with moderate to severe dementia and agitation significantly improved agitation, overall neuropsychiatric symptoms, and pain. These findings emphasise the importance of assessing and treating pain effectively as part of the overall treatment and prevention of agitation and aggression in patients with dementia. The results also highlight the potential value of effective treatment of pain as a key part of reducing the use of antipsychotics and other psychotropic drugs in residents of nursing homes.

The current study is the first adequately powered parallel group randomised controlled trial of pain management for the treatment of agitation in patients with moderate to severe dementia, and the positive response is supported by the worsening of agitation over the four weeks of follow-up after the intervention had stopped. At the end of the intervention the groups differed by 7.0 points, with a $17 \%$ advantage over control in the percentage change in score on the Cohen-Mansfield agitation inventory over the duration of the trial. To put this into context, the three studies using the Cohen-Mansfield agitation inventory as an outcome in randomised controlled trials of risperidone (the only licensed drug treatment for agitation or aggression in people with dementia) reported $3 \%, 13 \%$, and $18 \%$ advantages compared with placebo, respectively. ${ }^{25-27}$ The clinical significance of the benefit therefore compares well to the currently best available drug therapy. The clinical relevance is further supported by the significant correlation observed between change in agitation and change in pain. The results from previous open studies and case series are variable. A placebo controlled study of 167 patients in nursing homes, providing implementation of personalised, non-drug intervention, resulted in decreased agitation in the intervention group, ${ }^{21}$ but the findings were not replicated in an open study of 114 residents in nursing homes, which included pain management as part of a protocol to deal with unmet needs. ${ }^{13}$ A subsequent trial of pain treatment with paracetamol (acetaminophen) in 25 residents in 
nursing homes reported an increase in general activities and social interaction but no direct improvement in agitation. ${ }^{12}$ Finally, a double blind crossover trial of pain treatment with oxycodone and morphine in 47 residents in nursing homes, improved agitation in older but not in younger patients and showed a high frequency of dropouts. ${ }^{14}$ These studies show some support for the potential benefit of managing agitation by treating unmet needs such as pain, but with considerable differences in the type and magnitude of benefit, emphasising the need for a robust randomised controlled trial. ${ }^{11}$ The current, adequately powered parallel group randomised controlled trial shows more clear benefits. In clinical practice, by providing an effective treatment approach for people with dementia and agitation, improved management of pain should also help to reduce the number of prescriptions for antipsychotics in this population.

It is possible that agitation declined as a result of residents receiving sedation with opioid analgesics. However, only a few $(25.6 \%)$ were treated with sedative agents (table 2), and few residents $(n=3)$ were excluded because of drowsiness and nausea. Neither activities of daily living nor cognition worsened in the treatment group compared with control group, suggesting that sedation could not explain the reduction of agitation in the active group.

In this study, some behavioural symptoms improved in both intervention and control clusters. That may indicate a Hawthorne effect, ${ }^{28}$ perhaps related to factors such as increased staff training and support. Improvements in control groups is similar to other studies investigating drug and non-drug treatments for neuropsychiatric symptoms in people with dementia and is consistent with the potential benefits of interventions such as social interaction and reminiscence..$^{29}$ Precautions were taken to blind research assistants and caregivers to group allocation, but despite these efforts these studies will always be difficult to fully blind because of the requirements in a nursing home setting.

Importantly, in the current study, active intervention conferred significantly greater benefits over and above non-specific effects. The results highlight that a standardised approach to improved pain management is a practical intervention that would be straightforward to implement widely for the benefit of agitation in residents of nursing homes with dementia.

We thank the residents, their relatives, and nursing home staff for their willingness and motivation that made this study possible.

Contributors: BSH, CB, and DA conceived the study and obtained funding. All authors contributed to the study design, the carrying out of the study, and the writing of the manuscript. BSH and RS collected data. $\mathrm{OBN}$ and $\mathrm{BSH}$ contributed to the statistical analysis. BSH and DA are guarantors for the study.

Funding: This study was funded by the Norwegian Research Council (protocol code 189439), the University of Bergen (09/1568), and Kavli's Research Centre for Ageing and Dementia, Haraldsplass Diakonian Hospital, Bergen, Norway.

Competing interests: All authors have completed the ICMJE uniform disclosure form at www.icmje.org/coi_disclosure.pdf (available on request from the corresponding author) and declare: no support from any organisation for the submitted work; no financial relationships with any organisations that might have an interest in the submitted work in the previous three years; no other relationships or activities that could appear to have influenced the submitted work.

Ethical approval: This study was approved by the regional committee for medical ethics, western Norway (REK-Vest 248.08). Written consent was given by all participants who had sufficient capacity. If participants did not have the capacity to give consent, written assent was provided by the next of kin in accordance with the requirements of the research ethics committee and Norwegian law at the time of the study.

Data sharing: No additional data available.

Dartigues JF. Alzheimer's disease: a global challenge for the 21st century. Lancet Neurol 2009;8:1082-3.

2 Cohen-Mansfield J, Libin A. Verbal and physical non-aggressive agitated behaviors in elderly persons with dementia: robustness of syndromes. J Psychiatr Res 2005;39:325-32.

3 Testad I, Aasland AM, Aarsland D. Prevalence and correlates of disruptive behavior in patients in Norwegian nursing homes. Int J Geriatr Psychiatr 2007;22:916-21.

4 Rinaldi P, Spazzafumo L, Mastriforti R, Mattioli P, Marvardi M, Polidori MC, et al. Predictors of high level of burden and distress in caregivers of demented patients: results of an Italian multicenter study. Int J Geriatr Psychiatr 2005;20:168-74.

5 Selbaek G, Kirkevold O, Engedal K. The prevalence of psychiatric symptoms and behavioural disturbances and the use of psychotropic drugs in Norwegian nursing homes. Int J Geriatr Psychiatr 2007;22:843-9.

6 Barnerjee S. The use of antipsychotic medication for people with dementia. Time for action. A report for the Minister of State for Care Services. 2009. www.dh.gov.uk.

7 Husebo BS, Strand LI, Moe-Nilssen R, Husebo SB, Aarsland D, Ljunggren AE. Who suffers most? Dementia and pain in nursing home patients: a cross-sectional study. J Am Med Dir Assoc 2008;9:427-33.

8 Cohen-Mansfield J, Lipson S. The utility of pain assessment for analgesic use in persons with dementia. Pain 2008;134:16-23.

9 Mahoney AE, Peters $L$. The Mahoney pain scale: examining pain and agitation in advanced dementia. Am J Alzheimers Dis Other Demen 2008;23:250-61.

10 Lyketsos CG, Colenda CC, Beck C, Blank K, Doraiswamy MP, Kalunian DA, et al. Position statement of the American Association for Geriatric Psychiatry regarding principles of care for patients with dementia resulting from Alzheimer disease. Am J Geriatr Psychiatr 2006;14:561-73.

11 Husebo BS, Ballard C, Aarsland D. Pain treatment of agitation in patients with dementia: a systematic review. Int $J$ Geriatr Psychiatry 2011; published online 9 February.

12 Chibnall JT, Tait RC, Harman B, Luebbert RA. Effect of acetaminophen on behavior, well-being, and psychotropic medication use in nursing home residents with moderate-to-severe dementia. J Am Geriatr Soc 2005:53:1921-9.

13 Kovach CR, Logan BR, Noonan PE, Schlidt AM, Smerz J, Simpson M, et al. Effects of the Serial Trial Intervention on discomfort and behavior of nursing home residents with dementia. Am J Alzheimer Dis Other Demen 2006;21:147-55.

14 Manfredi PL, Breuer B, Wallenstein S, Stegmann M, Bottomley G, Libow L. Opioid treatment for agitation in patients with advanced dementia. Int $J$ Geriatr Psychiatr 2003;18:700-5.

15 Cohen-Mansfield J, Libin A. Assessment of agitation in elderly patients with dementia: correlations between informant rating and direct observation. Int $J$ Geriatr Psychiatr 2004;19:881-91.

16 Cummings JL, Aarsland D. Neuropsychiatric inventory NPI. Neurology 1994;44:2308-14

17 American Geriatric Society Panel. The management of chronic pain in older persons. J Am Geriatr Soc 1998;46:635-51.

18 Husebo BS, Strand LI, Moe-Nilssen R, Husebo SB, Ljunggren AE. Pain in older persons with severe dementia. Psychometric properties of the Mobilization-Observation-Behaviour-Intensity-Dementia (MOBID-2) Pain Scale in a clinical setting. Scand J Caring Sci 2010;24:380-91.

19 Folstein MF, Folstein SE, McHugh PR. Mini-mental state-practical method for grading cognitive state of patients for clinician. J Psychiatr Res 1975;12:189-98.

20 Sheikh K, Smith DS, Meade TW, Goldenberg E, Brennan PJ, Kinsella G. Repeatability and validity of a modified activities of daily living (ADL) index in studies of chronic disability. Int Rehab Med 1979;1:51-8.

21 Cohen-Mansfield J, Libin A, Marx MS. Nonpharmacological treatment of agitation: a controlled trial of systematic individualized intervention. J Gerontol Ser A Biol Sci Med Sci 2007;62:908-16.

22 Ballard C, Brown R, Fossey J, Douglas S, Bradley P, Hancock J, et al. Brief psychosocial therapy for the treatment of agitation in Alzheimer's disease (the CALM-AD trial). Am J Geriatr Psychiatry 2009;17:726-33.

23 Campbell MK, Elbourne DR, Altman DG. CONSORT statement: extension to cluster randomised trials. BMJ 2004;328:702-8.

24 Vickers AJ, Altman DG. Analysing controlled trials with baseline and follow up measurements. BMJ 2001;323:1123-4.

25 Deberdt WG, Dysken MW, Rappaport SA, Feldman PD, Young CA, Hay DP, et al. Comparison of olanzapine and risperidone in the treatment of psychosis and associated behavioral disturbances in patients with dementia. Am J Geriatr Psychiatry 2005:13:722-30.

26 Brodaty H, Ames D, Snowdon J, Woodward M, Kirwan J, Clarnette R, et al. A randomized placebo-controlled trial of risperidone for the treatment of aggression, agitation, and psychosis of dementia. J Clin Psychiatry 2003;64:134-43.

27 De Deyn PP, Rabheru K, Rasmussen A, Bocksberger JP, Dautzenberg PL, Eriksson S, et al. A randomized trial of risperidone, placebo, and haloperidol for behavioral symptoms of dementia. Neurology 1999;53:899-901.

28 McCarney R, Warner J, lliffe S, van Haselen R, Griffin M, Fisher P. The Hawthorne Effect: a randomised, controlled trial. BMC Med Res Methodol 2007;7:30.

29 Livingston G, Johnston K, Katona C, Paton J, Lyketsos CG. Systematic review of psychological approaches to the management of neuropsychiatric symptoms of dementia. Am J Psychiatr 2005;162:1996-2021.

Accepted: 14 May 2011 


\section{What is already known on this topic}

Many people with dementia have painful conditions, which in people with impaired language and abstract thinking may manifest as agitation

An estimated 180000 people with dementia in the United Kingdom are prescribed antipsychotics for agitation

\section{What this study adds}

A standardised protocol to treat pain in residents of nursing homes with moderate to severe dementia significantly improved agitation, aggression, and pain

Improved treatment of pain could help to reduce the unnecessary use of antipsychotics in people with dementia in nursing homes

Standardised assessment and treatment of pain should be an integral part of the clinical management pathway for people with dementia in nursing homes

\section{Tables}

\section{Table 1| Baseline personal and clinical characteristics of nursing home residents assigned to intervention (stepwise protocol for treatment} of pain) or continued usual medical care. Values are numbers (percentages) of patients unless stated otherwise

\begin{tabular}{|c|c|c|}
\hline Characteristics & Control group ( $n=177$ residents) & Intervention group ( $\mathrm{n}=175$ residents) \\
\hline No of clusters* & 27 & 33 \\
\hline Median (range) of patients per cluster & $7(3-10)$ & $6(2-10)$ \\
\hline Mean (range) age (years) & $87(67-104)$ & $85(65-101)$ \\
\hline Women & $131(74)$ & $132(75)$ \\
\hline \multicolumn{3}{|l|}{ Prescribed drugs: } \\
\hline Antipsychotics & $47(27)$ & $43(25)$ \\
\hline Anxiolytics or hypnotics & $88(50)$ & $80(46)$ \\
\hline Anti-dementia & $44(25)$ & $53(30)$ \\
\hline Opioid analgesics & $32(18)$ & $35(20)$ \\
\hline Peripheral analgesics & $71(40)$ & $75(43)$ \\
\hline Median (range) Cohen-Mansfield agitation inventory (scores 29-203) $\dagger$ & $51(39-114)$ & $53(39-126)$ \\
\hline Median (range) mini-mental state examination (scores $0-30$ ) $\ddagger$ & $8(0-20)$ & $7(0-20)$ \\
\hline Median (range) functional assessment staging (scores 1-7)§ & $6(4-7)$ & $6(4-7)$ \\
\hline Median (range) neuropsychiatric inventory—nursing home version (scores 1-144) & $29(0-97)$ & $32(1-101)$ \\
\hline Median (range) MOBID-2 pain scale $(\text { scores } 0-10)^{\star *}$; pain $\geq 3$ & $3.0(0-10) ; 98(55)$ & $4.0(0-10) ; 103(59)$ \\
\hline Median (range) activities of daily living (scores $0-20)+\dagger$ & $8.0(0-20)$ & $7.00(0-19)$ \\
\hline \multicolumn{3}{|l|}{ MOBID-2=mobilisation-observation-behaviour-intensity-dementia-2. } \\
\hline \multicolumn{3}{|l|}{${ }^{*}$ Cluster defined as a single independent nursing home unit. } \\
\hline \multicolumn{3}{|l|}{ †Higher scores indicate more agitation (scores $\geq 39$ usually accepted as clinically significant). } \\
\hline \multicolumn{3}{|l|}{ fHigher scores indicate more cognitive impairment. } \\
\hline \multicolumn{3}{|l|}{$\S$ Higher scores indicate more cognitive impairment. } \\
\hline \multicolumn{3}{|l|}{ \Higher scores indicate more agitation. } \\
\hline \multicolumn{3}{|l|}{${ }^{\star *} H i g h e r$ scores indicate more pain (scores $\geq 3$ accepted as clinically relevant). } \\
\hline ††Higher scores indicate more activities of daily living. & & \\
\hline
\end{tabular}


Table 2/ Stepwise protocol for treatment of pain

\begin{tabular}{|c|c|c|c|c|}
\hline Step & Pain treatment at baseline & Study treatment & Dosage & No $(\%)$ of residents $(n=175)$ \\
\hline 1 & No analgesics, or low dose of paracetamol & Paracetamol (acetaminophen) & Maximum dose $3 \mathrm{~g} /$ day & $120(69)^{*}$ \\
\hline 2 & $\begin{array}{l}\text { Full dose of paracetamol or low dose } \\
\text { morphine }\end{array}$ & Morphine & $\begin{array}{l}5 \mathrm{mg} \text { twice daily; maximum dose } 10 \mathrm{mg} \\
\text { twice daily }\end{array}$ & $4(2)$ \\
\hline 3 & $\begin{array}{l}\text { Low dose buprenorphine or inability to } \\
\text { swallow }\end{array}$ & Buprenorphine transdermal patch & $5 \mu \mathrm{g} / \mathrm{h}$, maximum dose $10 \mu \mathrm{g} / \mathrm{h}$ & $39(22) \dagger$ \\
\hline 4 & Neuropathic pain & Pregabaline & $\begin{array}{l}25 \mathrm{mg} \text { once daily; maximum dose } 300 \\
\mathrm{mg} / \text { day }\end{array}$ & $12(7)$ \\
\hline
\end{tabular}

*In nine participants an existing low dosage was increased.

†Dosage was increased in eight participants. 
Table 3| Comparison of Cohen-Mansfield agitation inventory (CMAI) total score between control and intervention (stepwise protocol for treatment of pain) groups using repeated measures analysis of covariance (ANCOVA)*

\begin{tabular}{|c|c|c|c|c|c|}
\hline \multirow[b]{2}{*}{ Week } & \multicolumn{2}{|c|}{ Mean (SD) CMAI total score } & \multicolumn{2}{|c|}{ Effect of intervention on CMAI total† } & \multirow{2}{*}{$\begin{array}{c}\text { Intracluster } \\
\text { correlation } \\
\text { coefficientł }\end{array}$} \\
\hline & Control group & Intervention group & Estimate $(95 \% \mathrm{Cl})$ & $P$ value & \\
\hline 0 & $56.2(16.1), \mathrm{n}=177$ & 56.5 (15.2), $\mathrm{n}=175$ & - & - & 0.162 \\
\hline 2 & $53.9(17.0), \mathrm{n}=161$ & 52.0 (19.5), $n=158$ & $-3.6(-0.5$ to -6.7$)$ & 0.022 & 0.261 \\
\hline 4 & 52.5 (16.3), $\mathrm{n}=160$ & $49.4(19.0), \mathrm{n}=148$ & $-4.1(-0.9$ to -7.4$)$ & 0.012 & 0.231 \\
\hline 8 & $52.8(16.8), \mathrm{n}=157$ & 46.9 (18.7), $n=147$ & $-7.0(-3.7$ to -10.3$)$ & $<0.001$ & 0.226 \\
\hline 12 & 52.5 (16.0), n=152 & 50.3 (20.3), $n=142$ & $-3.2(0.1$ to -6.4$)$ & 0.058 & 0.253 \\
\hline
\end{tabular}

*Baseline score as covariate and least squares weighted by number of patients within cluster; $P$ value from multivariate test of intervention was 0.002 , and cross effect between week and intervention was $<0.001$.

†Variable estimate by week of effect of intervention on CMAI score from estimated model.

$\ddagger$ Proportion of total variance between clusters, and measured within framework of ANCOVA. 
Table 4| Comparison of neuropsychiatric inventory-nursing home version (NPI-NH) total score between control and intervention (stepwise protocol for treatment of pain) groups using repeated measures analysis of covariance (ANCOVA)*

\begin{tabular}{|c|c|c|c|c|c|}
\hline \multirow[b]{2}{*}{ Week } & \multicolumn{2}{|c|}{ Mean (SD) NPI-NH total } & \multicolumn{2}{|c|}{ Effect of intervention on NPI-NH total† } & \multirow{2}{*}{$\begin{array}{l}\text { Intracluster } \\
\text { correlation } \\
\text { coefficient: }\end{array}$} \\
\hline & Control group & Intervention group & Estimate $(95 \% \mathrm{Cl})$ & $P$ value & \\
\hline$\underline{0}$ & $31.4(21.4), n=177$ & 34.8 (21.9), $\mathrm{n}=175$ & - & - & 0.106 \\
\hline 2 & 26.1 (19.2), $n=161$ & $26.5(20.3), \mathrm{n}=158$ & $-2.9(0.03$ to -5.9$)$ & 0.052 & 0.129 \\
\hline 4 & $26.0(20.1), n=160$ & $23.4(20.0), n=148$ & $-5.7(-2.3$ to -9.1$)$ & 0.001 & 0.116 \\
\hline 8 & $26.9(20.7), n=157$ & $21.0(19.3), n=147$ & $-9.0(-5.5$ to -12.6$)$ & $<0.001$ & 0.157 \\
\hline 12 & $28.0(21.1), n=152$ & $23.0(20.0), n=142$ & $-8.4(-4.7$ to -12.2$)$ & $<0.001$ & 0.210 \\
\hline
\end{tabular}

*Baseline score as covariate and least squares weighted by number of patients within cluster; $P$ values from multivariate test of intervention and cross effect between week and intervention were both $<0.001$.

†Variable estimate by week of effect of intervention on NPI-NH from estimated model.

$\ddagger$ Proportion of total variance between clusters, and measured within framework of ANCOVA. 
Table 5| Comparison of mobilisation-observation-behaviour-intensity-dementia-2 (MOBID-2) pain scale total score between control and intervention (stepwise protocol for treatment of pain) groups using repeated measures analysis of covariance (ANCOVA)*

\begin{tabular}{|c|c|c|c|c|c|}
\hline \multirow[b]{2}{*}{ Week } & \multicolumn{2}{|c|}{ Mean (SD) MOBID-2 total } & \multicolumn{2}{|c|}{ Effect of intervention on MOBID-2 total† } & \multirow{2}{*}{$\begin{array}{c}\text { Intracluster } \\
\text { correlation } \\
\text { coefficient }\end{array}$} \\
\hline & Control group & Intervention group & Estimate $(95 \% \mathrm{Cl})$ & $P$ value & \\
\hline 0 & $3.7(2.5), n=163$ & $3.8(2.7), n=164$ & & & 0.094 \\
\hline 2 & $3.5(2.4), n=159$ & $2.9(2.5), n=152$ & $-0.7(-0.4$ to -1.1$)$ & $<0.001$ & 0.070 \\
\hline 4 & $3.3(2.4), \mathrm{n}=155$ & $2.7(2.2), n=146$ & $-0.8(-0.4$ to -1.2$)$ & $<0.001$ & 0.059 \\
\hline 8 & $3.5(2.6), n=154$ & $2.3(2.1), n=145$ & $-1.3(-0.8$ to -1.7$)$ & $<0.001$ & 0.082 \\
\hline 12 & $3.5(2.5), n=151$ & $2.9(2.6), n=140$ & $-0.8(-0.3$ to -1.2$)$ & 0.001 & 0.139 \\
\hline
\end{tabular}

*Baseline score as covariate and least squares weighted by number of patients within cluster; $P$ value from multivariate test of intervention was $<0.001$, and cross effect between week and intervention was 0.009 .

†Variable estimate by week of effect of intervention on MOBID-2 from estimated model.

¥Proportion of total variance between clusters, and measured within framework of ANCOVA. 


\section{Figures}

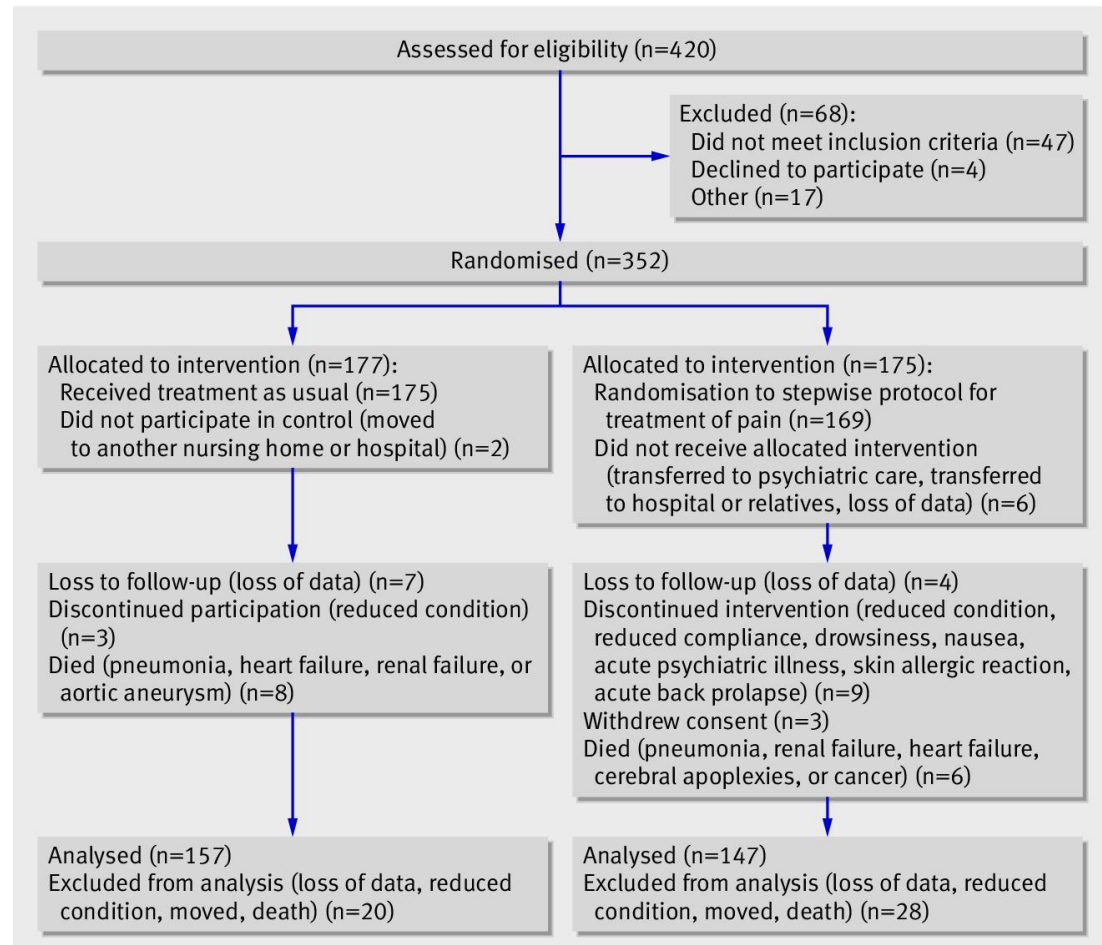

Fig 1 Flow of participants through trial

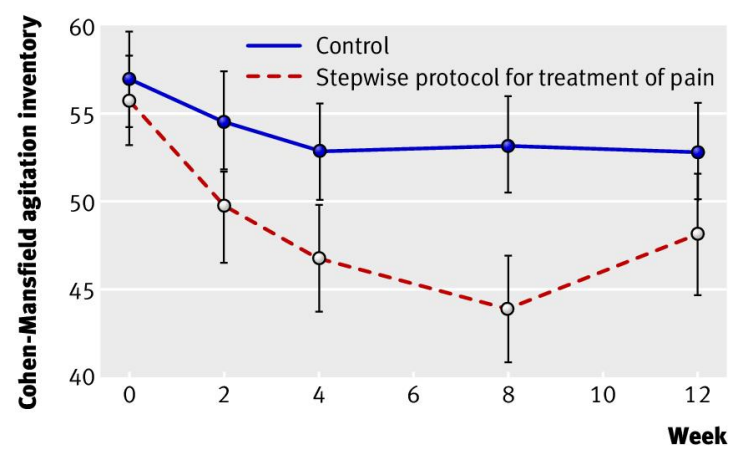

Fig 2 Cohen-Mansfield agitation inventory scores, with 95\% confidence intervals, over study period 